\title{
LA-UR-18-29104
}

Approved for public release; distribution is unlimited.

Title: $\quad$ End of Year Report for ICF Related Activities on J14C

Author(s): $\quad$ Gooden, Matthew Edgell

Intended for: $\quad$ Report

Issued: 
Disclaimer:

Los Alamos National Laboratory, an affirmative action/equal opportunity employer, is operated by the Los Alamos National Security, LLC for the National Nuclear Security Administration of the U.S. Department of Energy under contract DE-AC52-06NA25396. By approving this article, the publisher recognizes that the U.S. Government retains nonexclusive, royalty-free license to publish or reproduce the published form of this contribution, or to allow others to do so, for U.S. Government purposes. Los Alamos National Laboratory requests that the publisher identify this article as work performed under the auspices of the U.S. Department of Energy. Los Alamos National Laboratory strongly supports academic freedom and a researcher's right to publish; as an institution, however, the Laboratory does not endorse the viewpoint of a publication or guarantee its technical correctness. 


\section{End of Year Report for ICF Related Activities on J14C}

There were two main activities related to Radchem at NIF for FY'18: Reaction-In-Flight (RIF) neutron measurements and a Bromine-doped Capsule dedicated experiment. The RIF measurements were performed by neutron activation of Thulium and Bismuth foils, held at 50 $\mathrm{cm}$ from Target Chamber Center (TCC), in the Neutron Activation Detectors (NADs) attached to one or more Diagnostic Instrument Manipulators (DIMs). The Bromine-doped capsule experiment, which was to be a follow-on shot to one performed in Q4 of FY'17, was not shot during this FY.

\section{RIFS:}

The RIF experiments were carried out as 'ride-along' measurements on a number of shot platforms: HighFoot, High Density Carbon (HDC), BigFoot, etc. Thulium and Bismuth foils are collocated in a NAD which is attached to one of the 3 DIMs inside of the target chamber and are $\sim 50 \mathrm{~cm}$ from TCC. The activation disks of Thulium and Bismuth are each $50 \mathrm{~mm}$ in diameter and 0.5 and $5 \mathrm{~mm}$ thick, respectively.

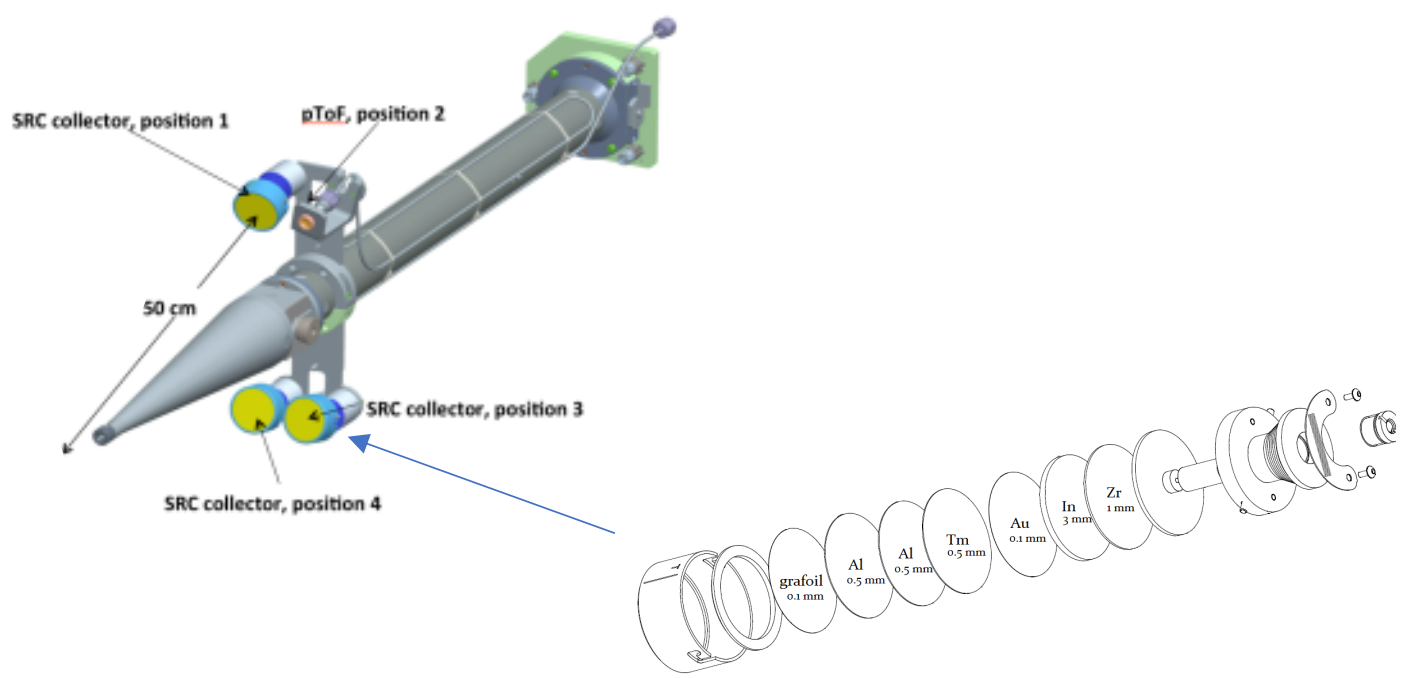

Figure 1: Diagram of DIM in the NIF target chamber, showing the placement of the activation foil NADs behind the Solid Radiochemistry Collectors (SRCs).

During this FY, we have been able to ride along on approximately 10 high-yield, cryogenic shots at NIF. Due to shot performance and technical challenges about 6 of those have produced data that is now being analyzed by T2. The Thulium foils are usually shipped back to LANL post shot for gamma spectroscopy on the LANL Count Room Clover Detector system, while the Bismuth disks remain at LLNL and are assayed on the sister system to the one at LANL. The HED Radchem team are free users of the LANL Count Room clover system and not owners of it, and it suffered a major failure in July 2017 that forced a return to the manufacturer for repair that 
kept the system inoperable for $\sim 9$ months. During this period the Thulium was kept at LLNL and both sets of activation foils were assayed on the sister system. This limited the time either could be counted and caused a reduction in the statistical accuracy of the results.

We are actively working on methods of analysis for the Bismuth data to improve the statistical accuracy of the results. The reaction that we rely on is the $209 \mathrm{Bi}(\mathrm{n}, 4 \mathrm{n}) 206 \mathrm{Bi}$ reaction, with a threshold of $22.5 \mathrm{MeV}$. The resulting activity is very low and greatly complicated by a very complex decay scheme. We are working to take advantage of this complex decay scheme as a signature of $\mathrm{Bi}-206$ and hope that it will yield improvement in the results. The data is acquired in 'List-Mode', meaning each event is tagged with a time and energy, and therefore the data can be 'replayed' and sorted in a number of different ways. Therefore, should a successful improvement in the analysis be made, both past and future data would be improved. Of course, as yields at NIF have increase our signal has improved as well.

T2 is currently processing data from approximately 6 shots over the course of the FY, with 1-2 more shot results to be delivered shortly for analysis. As the analysis is entailed and shots from similar campaigns treated the same, data is usually provided in batches to be analyzed together.

\section{Bromine-Doped Capsule:}

The first bromine-doped capsule shot was performed in early Q4 of FY ' 17 . The HED council did not approved at Tier-1 shot for FY '18 for the back-up capsule that is available. The council did approve the shot as Tier-2, however, the shot was not completed. We have been approved for this shot for $F Y^{\prime} 19$, sometime in Q3-Q4. 